\title{
A SLNR-Based Precoding Scheme for Multi-Stream Multicasting Systems
}

\author{
Jin Guang, Cao Bing
}

\begin{abstract}
The problem of wireless security for the downlink of multi-stream multicasting MIMO systems overheard by multiple multi-antenna eavesdroppers is investigated. We assume that the transmitter has perfect knowledge about the channel to desired users and the eavesdroppers. An efficient secure precoding scheme is devised that is based on signal-to-leakage-and-noise ratio (SLNR). The scheme maximizes the overall minimum SLNR of intended users subject to constrains on total available power and maximum allowable signal-to-interference-and noise radios (SINRs) of the eavesdroppers. Our design decouples the precoder design problem by using SLNR and advocates joint optimization of the precoding matrices and AN spatial distribution with lower computation complexity. Simulation and complexity comparison are provided to show the benefits of the proposed scheme.
\end{abstract}

Index Terms-Signal-to-leakage-and-noise ratio (SLNR),linear precoding, multi-stream multicast.

\section{INTRODUCTION}

W IRELESS multicast will support a variety of application such as group-oriented mobile commerce, distance education, and military command and control systems [1]. Its solutions are needed to exploit the idiosyncracies of wireless medium, in which the communication can be disrupted because of interference, noise or signal attenuation. Quality of service (QoS) and maxmin fair (MMF) designs for multicasting applications were first proposed in [2]. However, it was shown that the core problem is NP-hard. In [3], a relaxation techniques based on semidefinite programming (SDP) is used to achieve the high-quality approximate solutions. Furthermore, the problem of transmit beamforming for multicasting to multiple cochannel groups under QoS and MMF criteria was investigated in [4]. Unfortunately, many related works, such as the aforementioned, do not guarantee any kind of security.

On the other hand, physical layer security has caught growing attention recently in both information theory [5] and signal processing [6], as a viable, powerful alternative to blocking eavesdropping attempts in an open wireless medium. One of the meaningful ideas for physical layer security is that of artificial noise (AN) [7], in which a fraction of the transmit power is allocated to send artificially generated noise, to interfere the eavesdroppers. In [8], the multicasting beamforming problem in MISO systems with secrecy constraints for arbitrary number of users was researched. Then, a SINR-based transmit beamforming scheme was extended to the multigroup multicast scenario in [6]. However, the SINR metric couples all of the precoding weights, which makes that it is very difficult to obtain the solution, even though transforming the nonconvex optimization problem to a convex and tractable fashion, by recasting the problem as a semidefinite program (SDP).

Manuscript received June 15, 2012.

Jin Guang is with School of Vehicle Engineering, Zhengzhou Railway Vocational and Technical College, Zhengzhou, China(e-mail:jguang80@sina.com). Cao Bing is with Electronic Engineering Department, Zhengzhou Railway Vocational and Technical College, Zhengzhou, China.
In this paper, we pursue an alternative scheme for designing transmit precoding matrices and AN based on SLNR, which can transform a coupled optimization problem into a completely decoupled one [9] for multi-stream multicasting MIMO systems. The design goal is to reduce the complexity of original problem without much degradation in the performance over the optimal solution. To do this, we consider the problem of maximizing the minimum SLNR, received by any of the intended users irrespective of the multicast group they belong to, subject to constrains on total available power and maximum allowable SINRs of the eavesdroppers. Fortunately, the objective function is the generalized Rayleigh quotient for a Hermitian and positive semidefinite matrix and a Hermitian and positive definite matrix. So the precoding matrix can be obtained with considering power constraints. However, we cannot simply "scale up" the precoding matrix to satisfy the constraints as [9]. The reason is that, we herein deal with an interference scenario, and boosting one group precoding matrix also increases interference to other groups, which introduces a power control problem as well as. The optimization problem still is nonconvex. We show that it can be actually be solved in a convex and tractable fashion by using semidefinite relaxation (SDR), an approximation technique based on convex optimization [10]. The main goal is achieved using the proposed scheme and confirmed by simulation and complexity comparison.

\section{SECURITY MODEL AND PROBLEM FORMULATION}

Consider a downlink multiuser MIMO system comprising a transmitter, Alice, with $N$ antennas and $K$ users, Bobs, each equipped with $M$ receive antennas. The transmission is overheard by $J$ eavesdroppers, Eves, each equipped with $Q$ receive antennas. Let $\mathbf{H}_{k} \in \mathbb{C}^{M \times N}$ denotes the channel from Alice to Bob $k$. And $\overline{\mathbf{H}}_{k}=\left[\mathbf{H}_{1}^{H}, \cdots, \mathbf{H}_{k-1}^{H}, \mathbf{H}_{k+1}^{H}, \cdots, \mathbf{H}_{K}^{H}\right]^{H} \in \mathbb{C}^{(K-1) M \times N}$ represent the corresponding concatenated leakage channel for legitimate users. Let $\mathbf{G}_{j} \in \mathbb{C}^{Q \times N}$ denotes the channel from Alice to Eve $j$. And $\overline{\mathbf{G}}=\left[\mathbf{G}_{1}^{H}, \cdots, \mathbf{G}_{J}^{H}\right]^{H} \in \mathbb{C}^{J Q \times N}$ represent the corresponding concatenated leakage channel for eavesdroppers. A spatially uncorrelated flat Rayleigh fading channel is assume. The elements of $\mathbf{H}_{k}$ and $\mathbf{G}_{j}$ are all modeled as independent and identically distributed complex Gaussian variables with zero-mean and unit-variance. Furthermore, we assume $\mathbf{H}_{k}, \mathbf{G}_{j}, \overline{\mathbf{H}}_{k}$ and also $\overline{\mathbf{G}}$, have full rank with probability one.

Let there be a total of $1 \leq T \leq K$ multicast groups, $\mathbb{T}_{1}, \cdots, \mathbb{T}_{T}$, where $\mathbb{T}_{t}$ is the index set of the receivers participating in multicast group $t$, and $t \in\{1, \cdots, T\}$. In addition, $\mathbb{T}_{t} \cap \mathbb{T}_{l}=\emptyset, l \neq t, \cup_{t} \mathbb{T}_{t}=\{1, \cdots, K\}$. For guaranteeing physicallayer security, AN-aided transmit precoding is considered. In such a scheme, neglecting the time index for notational simplicity, the 
transmit vector $\mathbf{x} \in \mathbb{C}^{N \times 1}$ takes the structure

$$
\mathbf{x}=\sum_{t=1}^{T} \mathbf{W}_{t} \mathbf{s}_{t}+\mathbf{Z}
$$

where $\mathbf{s}_{t}$ is a $I \times 1$ data stream for the group $t$, which is assumed to be independent of one another. And $I \leq M$ is the number of data streams supported for group $t$ and is assumed equal for all the groups for simplicity. We also assume $E\left\{\mathbf{s}_{t} \mathbf{s}_{t}^{H}\right\}=\mathbf{I}_{I}$ without loss of generality. And $\mathbf{W}_{t} \in \mathbb{C}^{N \times I}$ is the $t$ th transmit precoding matrix, $\mathbf{Z}$ is the AN vector. We assume that $Z \sim \mathcal{C N}(0, \Sigma)$, where $\Sigma \succeq 0$ denotes the AN spatial convariance. Then, for given Bob $k$ and Eve $j$, the received signal vector can be written as

$$
\begin{aligned}
\mathbf{y}_{b, k} & =\mathbf{H}_{k} \sum_{t=1}^{T} \mathbf{W}_{t} \mathbf{s}_{t}+\mathbf{H}_{k} \mathbf{Z}+\mathbf{n}_{b, k}, k=1, \cdots, K \\
\mathbf{y}_{e, j} & =\mathbf{G}_{j} \sum_{t=1}^{T} \mathbf{W}_{t} \mathbf{s}_{t}+\mathbf{G}_{j} \mathbf{Z}+\mathbf{n}_{e, j}, j=1, \cdots, J
\end{aligned}
$$

where $\mathbf{n}_{b, k}$ and $\mathbf{n}_{e, j}$ are respectively complex circular Gaussian noise vector with zero-mean and positive definite covariance matrix $\mathrm{E}\left[\mathbf{n}_{b, k} \mathbf{n}_{b, k}{ }^{T}\right]=\sigma_{b}^{2} \mathbf{I}$ and $\mathrm{E}\left[\mathbf{n}_{e, j} \mathbf{n}_{e, j}{ }^{T}\right]=\sigma_{e}^{2} \mathbf{I}$. Let $\mathbf{R}_{\mathbf{H}, k}=$ $\mathbf{H}_{k}^{H} \mathbf{H}_{k}$ denotes the correlation matrix of $\mathbf{H}_{k}$. Following (2), the SINR of Bob $k$ can be formulated as

$$
\begin{aligned}
& S I N R_{b, k}\left(\Sigma, \mathbf{W}_{1}, \cdots, \mathbf{W}_{T}\right)= \\
& \frac{\operatorname{tr}\left(\mathbf{W}_{i}^{H} \mathbf{R}_{\mathbf{H}, k} \mathbf{W}_{i}\right)}{\sum_{t=1, t \neq i}^{T} \operatorname{tr}\left(\mathbf{W}_{t}^{H} \mathbf{R}_{\mathbf{H}, k} \mathbf{W}_{t}\right)+\operatorname{tr}\left(\Sigma \mathbf{R}_{\mathbf{H}, k}\right)+M / I \sigma_{b}^{2}}
\end{aligned}, k \in \mathbb{T}_{i}
$$

where $\operatorname{tr}(\cdot)$ denotes the trace operator. It is assumed that Eve can reduce the effects of multiuser interference. The SINR for Eve $j$ to eavesdrop the $i$ th data stream is

$$
\begin{aligned}
& S I N R_{e, j}\left(\Sigma, \mathbf{W}_{1}, \cdots, \mathbf{W}_{T}\right)= \\
& \frac{\operatorname{tr}\left(\mathbf{W}_{i}^{H} \mathbf{R}_{G, j} \mathbf{W}_{i}\right)}{\sum_{t=1, t \neq i}^{T} \operatorname{tr}\left(\mathbf{W}_{t}^{H} \mathbf{R}_{G, j} \mathbf{W}_{t}\right)+\operatorname{tr}\left(\Sigma \mathbf{R}_{G, j}\right)+Q / I \sigma_{e}^{2}}
\end{aligned} j \in\{1, \cdots, J\}
$$

By using the SINR-based precoding scheme with max-min-fair criterion for standard multigroup multicast in [4] [6], the design problem can be formulated as follows:

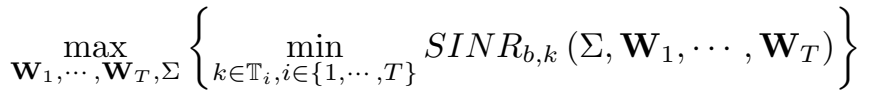

s.t. $S I N R_{e, j}\left(\Sigma, \mathbf{W}_{1}, \cdots, \mathbf{W}_{T}\right) \leq \gamma, j=1, \cdots, J, i=1, \cdots, T$

$$
\sum_{\substack{t=1 \\ \Sigma \succeq 0}}^{T} \operatorname{tr}\left(\mathbf{W}_{t} \mathbf{W}_{t}^{H}\right)+\operatorname{tr}(\Sigma) \leq P
$$

where $P$ is a given transmit total power, and $\gamma>0$ denotes the maximum allowable SINR threshold for Eves to eavesdrop any data stream. According to the Claim 2 in [4], we can proof problem (5) is NP-hard. Although the problem can be handled approximately by semidefinite relaxation (SDR) [6], the relaxation of problem (5) is still a quasi-convex problem. All in all, the computation complexity of solving problem (5) is very high, which includes bisection search, solving a sequence of SDPs and Gaussian randomization [10] and so on. For $T$ coupled matrix variables in objective function, the main computation complexity is introduced by the SDPs problem. Thus, in the following section, a suboptimal scheme is proposed based on SLNR [9], which can transform the coupled optimization problem into an approximate decoupled one.

\section{PROPOSED PRECODING SCHEME}

In [9], the SLNR is defined as the ratio of received signal power at the desired user to received signal power at other terminals plus noise power. Considering $J$ Eves, in multi-stream multicast MIMO system, the SLNR related to Bob $k$ can be given by

$$
\begin{aligned}
& S L N R_{b, k}\left(\mathbf{W}_{i}\right)= \\
& \frac{\operatorname{tr}\left(\mathbf{W}_{i}^{H} \mathbf{R}_{\mathbf{H}, k} \mathbf{W}_{i}\right)}{\operatorname{tr}\left(\mathbf{W}_{i}^{H}\left(\overline{\mathbf{H}}_{k}^{H} \overline{\mathbf{H}}_{k}+\overline{\mathbf{G}}^{H} \overline{\mathbf{G}}+M / I \sigma_{b}^{2} \mathbf{I}\right) \mathbf{W}_{i}\right)}, k \in \mathbb{T}_{i}
\end{aligned}
$$

According to the SLNR criterion and (6), the precoding matrix for the $i$ th group, $\mathbf{W}_{i}$, can be designed based on the following metric

$$
\mathbf{W}_{i}^{\text {opt }}=\arg \max _{\mathbf{W}_{i} \in C_{N \times I}} \min _{k \in T_{i}} S L N R_{b, k}\left(\mathbf{W}_{i}\right)
$$

Since $\mathbf{R}_{\mathbf{H}, k}$ is Hermitian and positive semidefinite (HPSD) and $\overline{\mathbf{H}}_{l}^{H} \overline{\mathbf{H}}_{l}+\overline{\mathbf{G}}^{H} \overline{\mathbf{G}}+M / I \sigma_{b}^{2} \mathbf{I}$ is Hermitian and positive definite (HPD). Therefore, by generalized eigenvalue decomposition, there exists an invertible matrix $\mathbf{F}_{k} \in \mathbb{C}^{N \times N}$ such that

$$
\begin{aligned}
& \mathbf{F}_{k}^{H} \mathbf{R}_{\mathbf{H}, k} \mathbf{F}_{k}=\Lambda_{i}=\operatorname{diag}\left(\lambda_{1}, \cdots, \lambda_{N}\right) \\
& \mathbf{F}_{k}^{H}\left(\overline{\mathbf{H}}_{k}^{H} \overline{\mathbf{H}}_{k}+\overline{\mathbf{G}}^{H} \overline{\mathbf{G}}+M / I \sigma_{b}^{2} \mathbf{I}\right) \mathbf{F}_{k}=\mathbf{I}_{N}
\end{aligned}
$$

with $\lambda_{1} \geq \lambda_{2} \geq \cdots \lambda_{N} \geq 0$. The $\left\{\lambda_{i}\right\}$ and the columns of $\mathbf{F}_{k}$ are the generalized eigenvalues and eigenvectors of the pair $\left\{\mathbf{R}_{\mathbf{H}, k}, \overline{\mathbf{H}}_{k}^{H} \overline{\mathbf{H}}_{k}+\overline{\mathbf{G}}^{H} \overline{\mathbf{G}}+M / I \sigma_{b}^{2} \mathbf{I}\right\}$.

Because (7) is a decoupled equality, we can get the optimal precoder matrix for each users by extracting the leading $I$ columns of $\mathbf{F}_{k}$ as $\mathbf{F}_{k}^{\text {opt }}=\rho_{k} \mathbf{F}_{k}\left(\mathbf{I}_{I} ; 0\right)$, where $\rho_{k}$ is a scaling factor for power constraint. Then the optimal precoder matrix for each group can be obtained by $\mathbf{W}_{i}^{o p t}=\mathbf{F}_{i_{k}}^{o p t}$, where $i_{k}=$ $\arg \min _{k \in \mathbb{T}_{i}} S L N R_{b, k}, i \in\{1, \cdots, T\}$. The interest, in the paper, is in active Eves cases, where Alice has knowledge of Eves' CSI. So the AN covariance can be designed to interfere Eves selectively [6]; ie., $\boldsymbol{\Sigma}=\beta \overline{\mathbf{G}}^{H} \mathbf{\mathbf { G }} /\|\overline{\mathbf{G}}\|^{2}$, where $\beta>0$ is a scale factor determining the power invested on AN. However, we cannot simply "scale up" the precoding matrix and AN covariance matrix to satisfy the constraints as [9]. The reason is that, we herein deal with an interference scenario, and boosting one group precoding matrix also increases interference to other groups, which introduces a power control problem as (9) which is at the top of the next page.

Although the resultant SDR problem is also a quasi-convex problem, its optimal solution can be obtained by applying a bisection search [10] in which a sequence of SDPs is solved. The complexity of the SDP problems, by introducing SLNR, will be reduced largely. The proposed scheme comprises the following steps

1) Step 1:

- Max-min SLNR criterion is used to model an optimization problem as seen in (7).

2) Step 2:

- Obtain SLNR-based candidate precoding matrices and AN covariance matrix.

3) Step 3:

- Model a power control problem (9) to get optimal power scale factors.

4) Step 4: 


$$
\begin{aligned}
& \max _{\rho_{1}, \cdots, \rho_{T}, \beta}\left\{\min _{k \in \mathbb{T}_{i}, i \in\{1, \cdots, T\}} \frac{\rho_{i}^{2} \operatorname{tr}\left(\left(\mathbf{F}_{i}\left(\mathbf{I}_{I} ; 0\right)\right)^{H} \mathbf{R}_{\mathbf{H}, k}\left(\mathbf{F}_{i}\left(\mathbf{I}_{I} ; 0\right)\right)\right)}{\sum_{t=1, t \neq i}^{T} \rho_{t}^{2} \operatorname{tr}\left(\left(\mathbf{F}_{t}\left(\mathbf{I}_{I} ; 0\right)\right)^{H} \mathbf{R}_{\mathbf{H}, k}\left(\mathbf{F}_{t}\left(\mathbf{I}_{I} ; 0\right)\right)\right)+\beta \operatorname{tr}\left(\overline{\mathbf{G}}^{H} \overline{\mathbf{G}} /\|\overline{\mathbf{G}}\|^{2} \mathbf{R}_{\mathbf{H}, k}\right)+M / I \sigma_{b}^{2}}\right\} \\
& \text { s.t. }
\end{aligned}
$$

- Use an available generalized linear-fractional programming method (e.g., by bisection) to solve problem (9). Get $\rho_{t}^{o p t}, t=0, \cdots, T$ and $\beta^{o p t}$.

5) Output:

- $\mathbf{W}_{t}^{\text {opt }}=\rho_{t}^{\text {opt }} F_{t}\left(\mathbf{I}_{I} ; 0\right), t=0, \cdots, T$ and $\Sigma^{o p t}=$
$\beta^{o p t} \overline{\mathbf{G}}^{H} \overline{\mathbf{G}} /\|\overline{\mathbf{G}}\|^{2}$

During step 4, a bisection search is used to handle the quasiconvex problem (9). Since in each iteration the interval is halved, the bisection search need $N_{B S}=\lceil\log 2(U-L) / \epsilon\rceil$ iterations [10].

\section{COMPLEXITY ANALYSIS}

Step 1-3 of proposed scheme requires constant time. In step 4 , the times of iteration is $N_{B S}=\lceil\log 2(U-L) / \epsilon\rceil$. In each iteration, a standard SDP problem is needed to be solved. The minimizing total power problem of (8) has $T+1$ matrix variables of size $N \times N, J$ linear coupled constraints and $K$ linear decoupled constraints. Interior point methods will take $\mathcal{O}\left(\sqrt{(T+1) N} \log _{2}(\varsigma)\right)$ iterations, with each iteration requiring $\mathcal{O}\left(4(T+1) N^{6}+(J+K)(T+1) N^{2}\right)$ arithmetic operations [4]. In step 5 , bisection search will be used repeatedly to obtain optimal $\alpha_{\mathbf{t}}^{\star}, \beta^{\star}, \mathbf{w}_{\mathbf{t}}^{\star}$ and $\chi^{\star}$. The times of iteration is $N_{B S}^{(R)}=\left[\log 2\left(U^{(R)}-L^{(R)}\right) / \epsilon^{(R)}\right]$. The problem has $T+1$ variable, $J$ linear coupled constraints and $K$ linear decoupled constraints. Similarly, interior point methods will take $\mathcal{O}\left(\sqrt{(T+1)} \log _{2}\left(\varsigma^{(R)}\right)\right)$ iterations, with each iteration requiring $\mathcal{O}(4(T+1)+(J+K)(T+1))$ arithmetic operations. Because no relationship with SLNR, the other arithmetic operations, such as matrix decomposition, multiplication as so on, are not considered in the paper.

Using the same analysis method, we can get the complexity of SINR-based precoding scheme. In the first bisection search, each iteration requiring $\mathcal{O}\left((T+1)^{3} N^{6}+(J+K)(T+1) N^{2}\right)$ arithmetic operations. In the stage of Gaussian randomization, each iteration requiring $\mathcal{O}\left((T+1)^{3}+(J+K)(T+1)\right)$ arithmetic operations. It is easily observed that the proposed scheme has a dramatic reduction in complexity compared to SINR-based precoding scheme, especially for large $T$.

\section{SIMULATION RESULTS}

The proposed algorithm is compared with [6], where a twogroup multicast transmission is considered. There are four Bobs

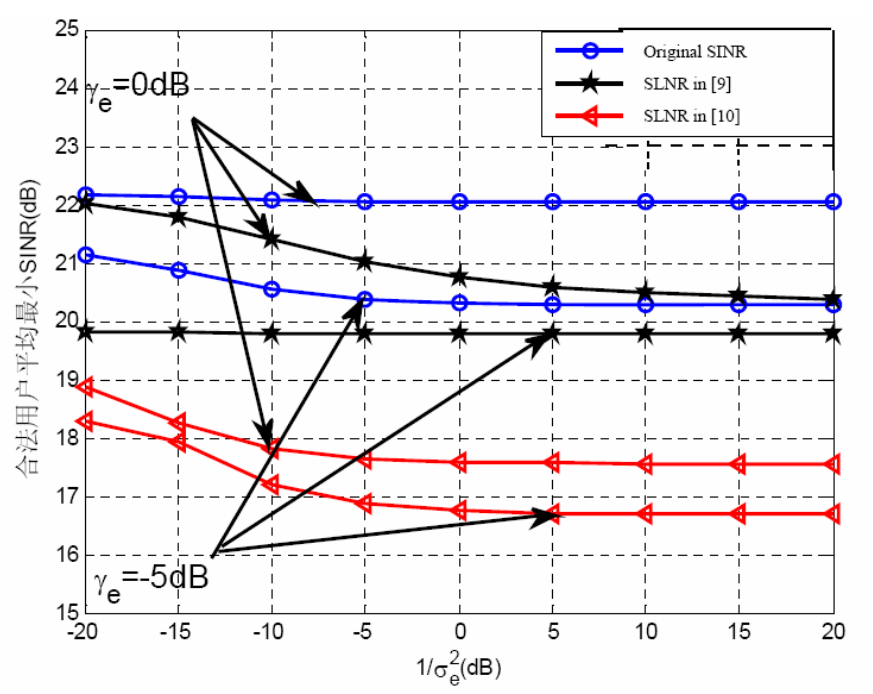

Fig. 1. Receiving performance comparison

and two Bobs form one group $\left(K=4, T=2, \mathbb{T}_{1}=\{1,2\}, \mathbb{T}_{2}=\right.$ $\{3,4\})$. Furthermore, the number of transmit antennas at Alice is $N=12$, and the number of antennas at Bobs are two $(M=2)$. There are four Eves, each equipped with two antenna $(J=4, Q=$ 2 ). The transmit power limit is $P=25 d B$ and the number of randomization is $L=30$. Bobs' noise power is assumed as $\sigma_{b}^{2}=$ $0 d B$, and the number of trials for Monte Carlo simulations is 1000 . In Fig.1, the SINR curves of the worst-Bob are plotted versus $1 / \sigma_{e}^{2}$, which denotes the overhearing ability of Eves. According to (4), the SINR can be calculated after the approximate solutions of (7) are available. It is observed that for the specification $\gamma=$ $0 d B$, the SINR gaps between the SINR-based scheme and the SLNR-based scheme are small and do not exceed $1 \mathrm{~dB}$. But, when applying a more stringent specification on Eves by setting $\gamma=$ $-5 d B$, we can get that.

\section{CONCLUSION}

In this paper, a new secure precoding scheme, which is based on SLNR and AN, for the downlink of multigroup multicast MIMO systems was proposed. The aim to provide a reasonable tradeoff between performance and computational complexity, is achieved using the proposed scheme and confirmed by simulation. 


\section{REFERENCES}

[1] U. Varshney, "Multicast over wireless networks," Communications of the ACM, vol. 45, pp. 31-37, Dec. 2002.

[2] N. D. Sidiropoulos, T. N. Davidson, and Z.-Q. Luo, "Transmit beamforming for physical layer multicasting," IEEE Trans. Signal Process., vol. 54, no. 6, pp. 2239-2251, Jun. 2006.

[3] E. Karipidis, N. D. Sidiropoulos, and Z.-Q. Luo, "Far-field multicast beamforming for uniform linear antenna arrays," IEEE Trans. Signal Process., vol. 55, no. 10, pp. 4916-4927, Oct. 2007.

[4] E. Karipidis, N. D. Sidiropoulos, and Z.-Q. Luo, "Quality of Service and MaxMin Fair Transmit Beamforming to Multiple Cochannel Multicast Groups,' IEEE Trans. Signal Process., vol. 56, no. 3, pp. 1268-1280, March 2008

[5] A. Khisti and G. W. Wornell, "Secure transmission with multiple antennas: The MIMOME wiretap channel," IEEE Trans. Inform. Theory, vol. 56, pp 5515-5532, Nov. 2010
[6] Wei-Cheng Liao, Tsung-Hui Chang, Wing-Kin Ma, and Chong-Yung Chi, "QoS-based transmit beamforming in the presence of eavesdroppers: An artificial-noise-aided approach," IEEE Trans. Signal Process, vol. 59, no.3, pp. 1202-1216, 2011.

[7] Xiangyun Zhou, Matthew R. McKay, "Secure transmission with artificial noise over fading channels: Achievable rate and optimal power allocation," IEEE Trans., wireless communication, vol. 59, no. 8, pp. 3831-3842, 2010.

[8] Eduard A. Jorswieck, "Beamforming in Interference Networks: Multicast, MISO IFC and Secrecy Capacity," Int. Zurich Seminar on Communications (IZS), March 3-5, 2010

[9] Mirette Sadek, Alireza Tarighat and Ali H. Sayed, "A Leakage-Based Precoding Scheme for Downlink Multi-User MIMO Channels," IEEE Trans., wireless communication, vol.6, no.5, pp.1711-1721, May, 2007.

[10] S. Boyd and L. Vandenberghe, Convex Optimization. Cambridge, UK:Cambridge University Press, 2004. 